\title{
Bloch Electrons in Constant Electric Field
}

\author{
F. Bentosela \\ Department of Physics, Princeton University, Princeton, NJ 08540, USA
}

\begin{abstract}
The motion of a conduction electron of a crystal in a constant electric field is studied. It is shown that the modulus of the wave function in $p$ representation is well approximated by a periodic function for times smaller than several hundred periods.
\end{abstract}

The aim of this paper is to study the evolution of a wave packet describing a Bloch electron under the influence of a constant electric field, as a first step to understand from first principles, the electrical conductivity of crystals.

Contrarily, to the free case, in which the momentum average of the particle grows linearly in time as we can easily see from the expression: $e^{-i\left(p^{2}+e \mathscr{E} x_{1}\right) t}$ $=e^{-i e^{2} \mathscr{E}^{2} t^{3} / 3} e^{-i e \mathscr{E} x_{1} t} e^{i e \mathscr{E} p t^{2}} e^{-i t p^{2}}$, we find that in the presence of a periodic potential, $V(x)$, the particle moves to a certain extent, periodically in the crystal.

More precisely, the probability the particle has a momentum in the infinitesimal volume $[\bar{p}, \bar{p}+d \bar{p}]$ is at times $t, t+T, t+2 T$, almost the same, as $\psi_{t}(\mathbf{p})$ can be divided in three parts $\psi_{t}(\mathbf{p})=\psi_{t}^{(1)}(\mathbf{p})+\psi_{t}^{(2)}(\mathbf{p})+\psi_{t}^{(3)}(\mathbf{p})$ and it exists $T=\left|\frac{2 \pi}{a_{1}}\right| \hbar \frac{1}{e \mathscr{E}}$ such that :

$$
\begin{aligned}
\left|\psi_{n T}^{(1)}(\mathbf{p})\right| & =\left|\psi_{0}^{(1)}(\mathbf{p})\right|, \quad n \in \mathbb{Z}^{+}, \\
\left\|\psi_{t}^{(2)}\right\| & \leqq v t, \\
\left\|\psi_{t}^{(3)}\right\| & \leqq \alpha .
\end{aligned}
$$

$\alpha$ and $v$ are such that $\alpha+v T$ is much smaller than 1 . This result confirms the heuristic approach of the problem, given by the solid state physicists $[1-3]$ when they suggest to replace the classical Newton law $\frac{d \mathbf{p}}{d t}=e \mathscr{E}$ by $\frac{d \mathbf{k}}{d t}=e \mathscr{E}$ where $\mathbf{k}$, the crystal momentum, is an element of a torus.

* On leave from Department of Physics, Luminy, Université d'Aix-Marseille II, and Centre de Physique Théorique, CNRS, Marseille, France 
Let us recall some general properties of the Bloch Hamiltonian $H_{B}=-\frac{\Delta}{2 M}$ $+V$ one can find in $[4-7,9]$. In the "p-representation" the action of the hamiltonian on a function $\psi \in L^{2}\left(\mathbb{R}^{3}, d^{3} p\right)$ is given by:

$$
\left(H_{B} \psi\right)(\mathbf{p})=\frac{\mathbf{p}^{2}}{2 M} \psi(\mathbf{p})+\sum_{\mathbf{K}} V_{\mathbf{K}} \psi(p-\mathbf{K})^{1},
$$

where $V_{\mathbf{K}}$ are the Fourier coefficients of $V(\mathbf{x}) \cdot\{V(\mathbf{x}+\mathbf{R})=V(\mathbf{x})$ if $\mathbf{R} \in \mathbb{L}$ (direct lattice). Calling $\mathbb{I}^{\perp}=\left\{\mathbf{K} \in \mathbb{R}^{3} \mid \mathbf{K} \cdot \mathbf{R}=2 \pi n, \forall \mathbf{R} \in \mathbb{L}\right\}$ the reciprocal lattice we have: $V(\mathbf{x})=\sum_{\mathbf{K} \in \mathbb{L}^{\perp}} V_{\mathbf{K}} e^{i \mathbf{K} \mathbf{x}} \cdot L^{2}\left(\mathbb{R}^{3}, d^{3} p\right)$ is isomorphic to $\int_{\mathbb{B}}^{\oplus} \ell^{2} d^{3} k$ where $\ell^{2}$ denotes the usual Hilbert space of sequences, and $\mathbb{B}$ the Brillouin zone $\left(\mathbb{B}=\left\{\mathbf{p} \in \mathbb{R}^{3} \mid \mathbf{p}\right.\right.$ is nearer to 0 than to any other point of $\left.\mathbb{L}^{\perp}\right\}$ ); a general point of $\mathbb{B}$ is called $\mathbf{k}$, a crystal momentum. Let $H(\mathbf{k})$ be the operator of $\ell^{2}:(H(\mathbf{k}) u)(\mathbf{K})=\frac{(\mathbf{k}+\mathbf{K})^{2}}{2 M} u(\mathbf{K})$

$+\sum_{\mathbf{K}^{\prime}} V_{\mathbf{K}^{\prime}} u\left(\mathbf{K}-\mathbf{K}^{\prime}\right)$. Then, $H_{\mathbb{B}}$ is unitarily equivalent to the direct integral $\int_{\mathbb{B}}^{\oplus} H(\mathbf{k}) d^{3} k$. $H(\mathbf{k})$ has a pure point spectrum: $E_{1}(\mathbf{k}), E_{2}(\mathbf{k}) \ldots E_{n}(\mathbf{k}), \ldots$ Calling $\left\{w_{1}^{\mathbf{k}}(\mathbf{K})\right\}$, $\left\{w_{2}^{\mathbf{k}}(\mathbf{K})\right\}, \ldots\left\{w_{n}^{\mathbf{k}}(\mathbf{K})\right\}$ the corresponding eigenvectors, we can construct, by varying $\mathbf{k}$ in $\mathbb{B}, w_{n} \in L^{2}\left(\mathbb{R}^{3}, d^{3} p\right)$ defined by:

$$
w_{n}(\mathbf{p}) \equiv w_{n}^{\mathbf{k}}(\mathbf{K}) \quad \text { if } \quad \mathbf{p}=\mathbf{k}+\mathbf{K} .
$$

Writing $E_{n}(\mathbf{p}) \equiv E_{n}(\mathbf{k})$ if $\mathbf{p}=\mathbf{k}+\mathbf{K}$, we get :

$$
\left(H_{\mathbb{B}} w_{n}\right)(\mathbf{p})=\frac{\mathbf{p}^{2}}{2 M} w_{n}(\mathbf{p})+\sum_{\mathbf{K}} V_{\mathbf{K}} w_{n}(\mathbf{p}-\mathbf{K})=E_{n}(\mathbf{p}) w_{n}(\mathbf{p}) .
$$

It can be easily seen [6] that $w_{n}$ are the Fourier transforms of the so-called Wannier functions.

This "Wannier function" permits us to construct an unitary transformation between $L^{2}\left(\mathbb{R}^{3}\right)$ and $L^{2}(N \times B)$ given by:

$$
\begin{aligned}
U_{\mathbb{B}}: \psi(\mathbf{p}) \rightarrow a_{n}(\mathbf{p}) & =\sum_{\mathbf{K}} \overline{w_{n}(\mathbf{p}+\mathbf{K})} \psi(\mathbf{p}+\mathbf{K}), \\
\mathrm{U}_{\mathbb{B}}^{-1}: a_{n}(\mathbf{p}) \rightarrow \psi(p) & =\sum_{n} a_{n}(\mathbf{p}) w_{n}(\mathbf{p}) .
\end{aligned}
$$

To study the evolution of a particle under the semi-group generated by $H \equiv H_{\mathbb{B}}$ $+e \mathscr{E} x_{1}$ (where $e \mathscr{E} x_{1}$ is the potential associated to the electrical field $\mathscr{E}$ ) we will use the Trotter formula:

$$
e^{i(A+B) t}=S-\lim _{m \rightarrow \infty}\left[e^{i B t / m} \cdot e^{i A t / m}\right]^{m} .
$$

1 To simplify the text we will use the same characters to denote the operators in the " $\mathrm{x}$ " and in the "p-representation" 
There are several possible choices for $A$ and $B$. The most natural one is to take: $A=H_{\mathbb{B}}$ and $B=e \mathscr{E} x_{1}$ but it leads to a constant $v$ in formula (2) which is such that $v T$ is of the order of 1 ; then we cannot conclude that the particle motion is quite periodic.

We will split $x_{1}$ into two parts: $x_{1}=x_{1}^{\prime}+x_{1}^{\prime \prime}$ and take $A=H_{\mathbb{B}}+e \mathscr{E} x_{1}^{\prime}$ and $B$ $=e \mathscr{E} x_{1}^{\prime \prime}, x_{1}^{\prime}$ will be the multiplication by the periodic function which is equal to $x_{1}$ in the first unit cell.

Lemma 1. The operator $H^{\prime} \equiv H_{\mathbb{B}}+e \mathscr{E} x_{1}^{\prime}$ has analogous properties as $H_{\mathbb{B}}$. Calling $H^{\prime}(\mathbf{k}) \equiv H(\mathbf{k})+e \mathscr{E} x_{1}^{\prime}(\mathbf{k}), H^{\prime}$ is unitarily equivalent to

$$
\int_{\mathbb{B}}^{\oplus} H^{\prime}(\mathbf{k}) d^{3} k .
$$

Each of the $H^{\prime}(k)$ has a discrete spectrum.

Proof. As we know that $(H(\mathbf{k})-\zeta)^{-1}$ is a compact operator [4]. $\left(H^{\prime}(\mathbf{k})-\zeta\right)^{-1}$ $=(H(\mathbf{k})-\zeta)^{-1}-\left(H^{\prime}(\mathbf{k})-\zeta\right)^{-1} e \mathscr{E} x_{1}^{\prime}(\mathbf{k})(H(\mathbf{k})-\zeta)^{-1}$ is also compact, then $H^{\prime}(\mathbf{k})$ has a pure discrete spectrum.

Let us call $w_{n}^{\prime}$ the "Wannier functions" associated with $H^{\prime}$; any function $f e L^{2}\left(\mathbb{R}^{3}\right)$ can be decomposed on $w_{n}^{\prime}: f(\mathbf{p})=\sum_{n} a_{n}^{\prime}(\mathbf{p}) w_{n}^{\prime}(\mathbf{p})$ with, $a_{n}^{\prime}(\mathbf{p})=\sum_{\mathbf{K}} \overline{w_{n}^{\prime}(\mathbf{p}+\mathbf{K})}$ $f(\mathbf{p}+\mathbf{K})$.

We want to study the evolution of a wave packet which belongs to $P_{c} L^{2}\left(\mathbb{R}^{3}\right)$, at time $t=0$. (" $c$ " refers to a special band called the conduction band, which is the first not entirely filled band, see [1]); $P_{c}=\int_{\mathbb{B}}^{\oplus} P_{c}(\mathbf{k}) d^{3} k$. Call $P_{c}^{\prime}$ the analogous operator corresponding to the modified conduction band, i.e., to the eigenvalues of $H^{\prime}(\mathbf{k})$, $E_{c}^{\prime}(\mathbf{k})$, which are derived from $E_{c}(\mathbf{k})$ when we apply the perturbing term $e \mathscr{E} x_{1}^{\prime}(\mathbf{k})$ to $H_{\mathbb{B}}(\mathbf{k})$. Then $\psi \in P_{c} L^{2}\left(\mathbb{R}^{3}\right)$ can be written in the form

$$
\psi=P_{c}^{\prime} \psi+\left(1-P_{c}^{\prime}\right) \psi \text {. }
$$

We consider first the evolution of $P_{c}^{\prime} \psi$ in Theorems 1 and 2 and give an estimate of $\left\|\psi_{t}^{(3)}\right\| \equiv\left\|\mathrm{e}^{-i H t}\left(1-P_{c}^{\prime}\right) \psi\right\|=\left\|\left(1-P_{c}^{\prime}\right) \psi\right\|$ in Theorem 3 .

Theorem 1. Suppose the eigenvalues, $E_{c}^{\prime}(\mathbf{k})$, of $H^{\prime}(\mathbf{k})$ are non-degenerate ${ }^{2}$ then

$$
\psi_{t}^{(1)}(\mathbf{p})=\lim _{m \rightarrow \infty}\left[P_{c}^{\prime} e^{-i e \mathscr{E} x_{1}^{\prime \prime} t / m} e^{-i\left(H_{B}+e \mathscr{8} x_{1}^{\prime}\right) t / m} P_{c}^{\prime}\right]^{m} \psi
$$

is such that for almost every $p$ :

$$
\left|\psi_{n T}^{(1)}(\mathbf{p})\right|=\left|\psi_{0}^{(1)}(\mathbf{p})\right| \quad \text { with } \quad T=\frac{2 \pi}{\left|a_{1}\right|} \hbar \frac{1}{e \mathscr{E}}, \quad n \in \mathbb{Z}^{+} .
$$

2 This assumption guarantees that $w_{c}^{\prime}$ is an analytic function of the variable $\mathbf{p}$. If one starts with a conduction band separated by gaps from the others, as it occurs practically often; for sufficiently small $\mathscr{E}$, considering $e \mathscr{E} x_{1}^{\prime}$ as perturbation, we get the same property for the modified conduction band, $E_{c}^{\prime}(\mathbf{p})$. (In [10] one can find some sufficient conditions on the periodical potential to have a non-degenerate lowest band; see also [8]). 
Proof. The way of proving the result is simply do to an explicit calculation of $\left[P_{c}^{\prime} e^{-i e \delta x^{\prime} i t / m} e^{-i\left(H_{B}+e \delta x^{\prime}\right) t / m} P_{c}^{\prime}\right]^{m}$ and of its limit, when applied to $P_{c}^{\prime} \psi$ :

$$
\left(P_{c}^{\prime} \psi\right)(p)=a_{c}^{\prime}(\mathbf{p}) w_{c}^{\prime}(\mathbf{p}) \stackrel{e^{-\imath\left(H_{B}+e \delta \chi_{j}^{\prime}\right) t / m}}{\longrightarrow} a_{c}^{\prime}(\mathbf{p}) e^{-i E_{c}^{\prime}(\mathbf{p}) t / m} w_{c}^{\prime}(\mathbf{p}) .
$$

As $x_{1}^{\prime}$ and $x_{1}$ commute

$$
e^{-i e \sigma x_{1}^{\prime} t / m}=e^{-i e \mathscr{E} x_{1} t / m} e^{i e \mathscr{E} x_{1}^{\prime} t / m}
$$

$$
a_{c}^{\prime}(\mathbf{p}) e^{-i E_{c}^{\prime}(\mathbf{p}) \cdot t / m} w_{c}^{\prime}(\mathbf{p}) \stackrel{e^{i e \delta x_{1}^{\prime} t / m}}{\longrightarrow} a_{c}^{\prime}(\mathbf{p}) e^{-i E_{c}^{\prime}(\mathbf{p}) t / m}\left(e^{i e \delta x^{\prime} t t / m} w_{c}^{\prime}\right)(\mathbf{p})
$$$$
\stackrel{e^{-\imath e \mathscr{\delta} x_{1} t / m} \longrightarrow}{\longrightarrow} a_{c}^{\prime}(\mathbf{p}+e \mathscr{E} t / m) e^{-i E_{c}^{\prime}(\mathbf{p}+e \mathscr{E} t / m) \cdot t / m}\left(e^{-i e \mathscr{E} x_{1}^{\prime \prime} t / m} w_{c}^{\prime}\right)(\mathbf{p})
$$$$
\stackrel{P_{c}^{\prime}}{\longrightarrow} a_{c}^{\prime}(\mathbf{p}+e \mathscr{E} t / m) e^{-i E_{c}^{\prime}(\mathbf{p}+e \mathscr{E} t / m) \cdot t / m}\left[\sum_{\mathbf{K}} \overline{w_{c}^{\prime}(\mathbf{p}+\mathbf{K})}\left(e^{-i e \mathscr{E} x_{1}^{\prime \prime} t / m} w_{c}^{\prime}\right)(\mathbf{p}+\mathbf{K})\right] w_{c}^{\prime}(\mathbf{p}) \text {. }
$$

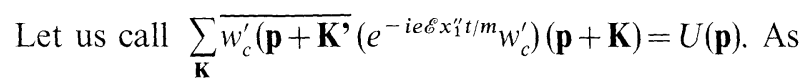

$$
\begin{aligned}
S_{m} \psi & \equiv P_{c}^{\prime} e^{-i e \mathscr{\delta} x_{1}^{\prime \prime} t / m} e^{-i\left(H_{B}+e \delta x^{\prime}\right) \cdot t / m} P_{c}^{\prime} \psi \\
& =a_{c}^{\prime}(\mathbf{p}+e \mathscr{E} t / m) e^{-i E_{c}^{\prime}(\mathbf{p}+e \delta t / m) \cdot t / m} U(\mathbf{p}) \cdot w_{c}^{\prime}(\mathbf{p}),
\end{aligned}
$$

we get:

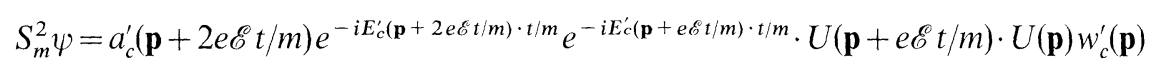

and

$$
\begin{aligned}
S_{m}^{m} \psi= & a_{c}^{\prime}(\mathbf{p}+e \mathscr{E} t) e^{-i E_{c}^{\prime}(\mathbf{p}+e \delta \mathscr{\delta} t) \cdot t / m} \ldots e^{-i E_{c}^{\prime}\left(\mathbf{p}+\frac{j}{m} e \mathscr{E} t\right) \cdot t / m} \ldots e^{-i E_{c}^{\prime}(\mathbf{p}+e \delta t / m) \cdot t / m} \\
& \cdot U\left(\mathbf{p}+\frac{m-1}{m} e \mathscr{E} t\right) \ldots U\left(\mathbf{p}+\frac{j-1}{m} e \mathscr{E} t\right) \ldots U(\mathbf{p}) \cdot w_{c}^{\prime}(\mathbf{p}) .
\end{aligned}
$$

As $a_{c}^{\prime}(\mathbf{p}+e \mathscr{E} T)=a_{c}^{\prime}(\mathbf{p})$, to end the proof we have to show that:

$$
\left|\lim _{m \rightarrow \infty} \prod_{j=1}^{m} e^{-i E_{c}^{\prime}\left(\mathbf{p}+\frac{j}{m} e \mathscr{E} t\right) \cdot t / m} U\left(\mathbf{p}+\frac{j-1}{m} e \mathscr{E} t\right)\right|=1 .
$$

The limit of

$$
\prod_{j=1}^{m} e^{-i E_{c}^{\prime}\left(\mathbf{p}+\frac{j}{m} e \delta t\right) \cdot t / m}=e^{-\frac{i}{e \delta_{j}} \sum_{j}^{m} E_{c}^{\prime}\left(\mathbf{p}+\frac{j}{m} e \delta t\right) \frac{e \delta t}{m}}
$$

as $m \rightarrow \infty$ is

$$
e^{-\frac{i}{e \delta} \int_{\mathbf{p}}^{\mathbf{p}+e \delta t} E_{c}^{\prime}\left(\mathbf{p}^{\prime}\right) d^{3} p^{\prime}} .
$$

Consider now :

$$
\log \prod_{j=1}^{m} U\left(\mathbf{p}+\frac{j-1}{m} e \mathscr{E} t\right) .
$$


Writing

$$
\begin{aligned}
& e^{-i e \mathscr{E} x_{1}^{\prime \prime} t / m}=1-i e \mathscr{E} x_{1}^{\prime \prime} t / m-\frac{(e \mathscr{E} t / m)^{2}}{2} \theta_{2}^{2}\left(x_{1}\right) x_{1}^{\prime \prime 2} \\
& +i \frac{(e \mathscr{E} t / m)^{3}}{3 !} \theta_{3}^{3}\left(x_{1}\right) x_{1}^{\prime \prime 3} ;\left\|\theta_{2}\right\|,\left\|\theta_{3}\right\| \leqq 1 \\
& U\left(\mathbf{p}+\frac{j-1}{m} e \mathscr{E} t\right) \\
& =1-i e \mathscr{E} t / m \sum_{\mathbf{K}} \overline{w_{c}^{\prime}\left(\mathbf{p}+\frac{j-1}{m} e \mathscr{E} t+\mathbf{K}\right)}\left[x^{\prime \prime} w_{c}^{\prime}\right]\left(p+\frac{j-1}{m} e \mathscr{E} t+\mathbf{K}\right), \\
& -\frac{1}{2}(e \mathscr{E} t / m)^{2} \sum_{\mathbf{K}} \overline{w_{c}^{\prime}\left(p+\frac{j-1}{m} e \mathscr{E} t+\mathbf{K}\right)}\left[\theta_{2}^{2} x_{1}^{\prime \prime 2} w_{c}^{\prime}\right]\left(\mathbf{p}+\frac{j-1}{m} e \mathscr{E} t+\mathbf{K}\right) \\
& +\frac{i}{3 !}(e \mathscr{E} t / m)^{3} \sum_{\mathbf{K}} \overline{w_{c}^{\prime}\left(p+\frac{j-1}{m} e \mathscr{E} t+\mathbf{K}\right)}\left[\theta_{3}^{3} x_{1}^{\prime \prime} w_{c}^{\prime}\right]\left(p+\frac{j-1}{m} e \mathscr{E} t+\mathbf{K}\right) \\
& \equiv 1+Y_{j} \text {. }
\end{aligned}
$$

Then

$$
\log \prod_{j=1}^{m} U\left(\mathbf{p}+\frac{j-1}{m} e \mathscr{E} t\right)=\sum_{j=1}^{m} \log \left(1+Y_{j}\right)=\sum_{j=1}^{m} Y_{j}-\frac{Y_{j}^{2}}{2} \frac{1}{\left(1+\theta^{\prime} Y_{j}\right)^{2}} ; 0<\theta^{\prime}<1 .
$$

The coefficient of $\left(\frac{e \mathscr{E} t}{m}\right)^{2}$ in this expression is bounded above, in fact $w_{c}^{\prime}$ is analytic so it belongs to the domains of $x_{1}^{\prime \prime}, x_{1}^{\prime \prime 2}=x_{1}^{2}-2 x_{1} x_{1}^{\prime}+x_{1}^{\prime 2}$ and $x_{1}^{\prime \prime 3}=x_{1}^{2}-3 x_{1}^{2} x^{\prime}+3 x_{1} x_{1}^{\prime 2}$ $-x_{1}^{\prime 3}$; by the same reason the coefficients of $\left(\frac{e \mathscr{E} t}{m}\right)^{s}, s=3,4,5,6$ are also bounded above, then:

$$
\lim _{m \rightarrow \infty} \sum_{j=1}^{m} \log \left(1+Y_{j}\right)=\lim _{m \rightarrow \infty} \sum_{j=1}^{m} Y_{j}=-i \int_{\mathbf{p}}^{\mathbf{p}+e \mathscr{E} t} \sum_{\mathbf{K}} \overline{w_{c}^{\prime}\left(\mathbf{p}^{\prime}+\mathbf{K}\right)}\left[x_{1}^{\prime \prime} w_{c}^{\prime}\right]\left(\mathbf{p}^{\prime}+\mathbf{K}\right) d^{3} p^{\prime} .
$$

We get finally:

$$
\begin{aligned}
& \lim _{m \rightarrow \infty}\left[P_{c}^{\prime} e^{-i e \mathscr{E} x_{1}^{\prime \prime} t / m} e^{-i\left(H+e \mathscr{E} x_{1}^{\prime}\right) \cdot t / m} P_{c}^{\prime}\right]^{m} \psi(\mathbf{p})
\end{aligned}
$$

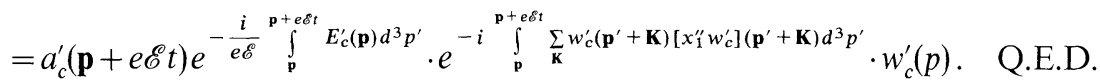

Let us now introduce the following notations

$$
\begin{aligned}
& S_{m} \equiv P_{c}^{\prime} e^{-i e \mathscr{E} x_{1}^{\prime \prime} t / m} e^{-i\left(H_{B}+e \mathscr{E} x_{1}^{\prime}\right) t / m} P_{c}^{\prime}, \\
& T_{m} \equiv\left(1-P_{c}^{\prime}\right) \ldots \ldots \ldots \ldots \ldots \ldots \ldots\left(1-P_{c}^{\prime}\right) \text {, } \\
& I_{m}^{\dagger} \equiv\left(1-P_{c}^{\prime}\right) \ldots \ldots \ldots \ldots \ldots \ldots P_{c}^{\prime} \text {, } \\
& I_{m}^{\downarrow} \equiv P_{c}^{\prime} \ldots \ldots \ldots \ldots \ldots \ldots \ldots . \ldots . \ldots . \ldots . . .
\end{aligned}
$$


Then we have by the Trotter formula:

$$
\psi_{t}-\psi_{t}^{(3)}=\mathrm{e}^{-i H t} P_{c}^{\prime} \psi=S-\lim _{m \rightarrow \infty}\left[S_{m}+T_{m}+I_{m}^{\uparrow}+I_{m}^{\downarrow}\right]^{m} P_{c}^{\prime} \psi .
$$

Recall that $\psi_{t}^{(1)}=s-\lim _{m \rightarrow \infty} S_{m}^{m} \psi$, now we want to prove that $\psi_{t}^{(2)}=\psi_{t}-\psi_{t}^{(1)}-\psi_{t}^{(3)}$ grows slowly in time.

Theorem 2. Suppose the conduction band is separated from the others by a gap, $\Delta E_{c}$, and $\mathscr{E}<\frac{\Delta E_{c}}{e\left|\mathbf{a}_{1}\right|}$, then $\left\|\psi_{t}-\psi_{t}^{(1)}-\psi_{t}^{(3)}\right\| \leqq v t, \forall t<\frac{1}{v}$, where $v$ depends essentially on $\mathscr{E}$ and on $\Delta E_{c}$.

Proof. Expanding $\left(S_{m}+T_{m}+I_{m}^{\uparrow}+I_{m}^{\uparrow}\right)^{m} P_{c}^{\prime}$ we get terms which can be represented by diagrams, for instance,

$$
S_{m}^{j_{3}} I_{m}^{\downarrow} T_{m}^{j_{2}} I_{m}^{\dagger} S_{m}^{j_{1}}\left(j_{1}+j_{2}+j_{3}=m-2\right) \text { will be represented by: }
$$

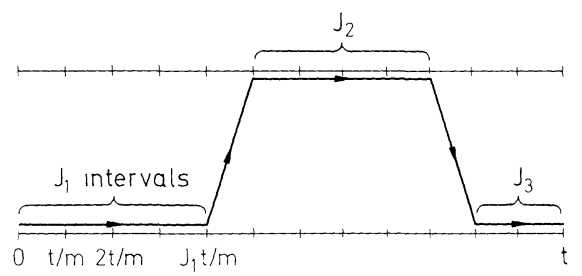

As $\left\|S_{m}^{j_{3}} I_{m}^{\downarrow} T_{m}^{j_{2}} I_{m}^{\uparrow} S_{m}^{j_{1}}\right\| \leqq\left\|I_{m}^{\downarrow}\right\| \cdot\left\|I_{m}^{\uparrow}\right\|$ to get an upper bound for $\left\|\left(S_{m}+T_{m}+I_{m}^{\uparrow}+I_{m}^{\downarrow}\right)^{m}-S_{m}^{m}\right\|$ it suffices to count the number of diagrams.

with 1 "up"

with 1 "up" and 1 "down"

with 2 "up" and 1 "down"

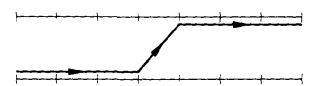

: $m$

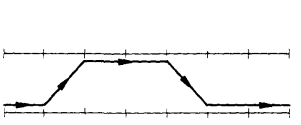

: $\left(\begin{array}{c}\mathrm{m} \\ 2\end{array}\right)$

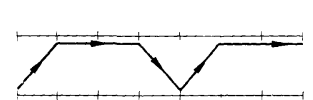

: $\left(\begin{array}{l}m \\ 3\end{array}\right)$

with $\ell$ "up" and $\ell-1$ "down"

$:\left(\begin{array}{c}\mathrm{m} \\ 2 \ell-1\end{array}\right)$

with $\ell$ "up" and $\ell$ "down"

Then

$$
\begin{aligned}
& \left\|\left(S_{m}+T_{m}+I_{m}^{\uparrow}+I_{m}^{\downarrow}\right)^{m} P_{c}^{\prime}-S_{m}^{m}\right\| \leqq m\left\|I_{m}^{\uparrow}\right\|+\left(\begin{array}{c}
m \\
2
\end{array}\right)\left\|I_{m}^{\uparrow}\right\|\left\|I_{m}^{\downarrow}\right\|+\ldots \\
& \left(\begin{array}{c}
m \\
2 \ell-1
\end{array}\right)\left\|I_{m}^{\downarrow}\right\|^{\ell-1}\left\|I_{m}^{\uparrow}\right\|^{\ell}+\ldots+\left(\begin{array}{c}
m \\
2 \ell
\end{array}\right)\left\|I_{m}^{\uparrow}\right\|^{\ell}\left\|I_{m}^{\downarrow}\right\|^{\ell}+\ldots+\left\|I_{m}^{\uparrow}\right\|^{m / 2}\left\|I_{m}^{\downarrow}\right\|^{m / 2} .
\end{aligned}
$$


Let $\mathscr{I}_{m}=\max \left(\left\|I_{m}^{\uparrow}\right\|,\left\|I_{m}^{\downarrow}\right\|\right)$. We see that

$$
\left\|\left(S_{m}+T_{m}+I_{m}^{\uparrow}+I_{m}^{\downarrow}\right)^{m} P_{c}^{\prime}-S_{m}^{m}\right\| \leqq\left(1+\mathscr{I}_{m}\right)^{m}-1 .
$$

Now the idea is to prove that $\mathscr{I}_{m} \leqq \frac{v}{2} \frac{t}{m}$, then for all $t$ such that $v t<1$ we conclude that:

$$
\left\|\psi_{(t)}-\psi_{(t)}^{(1)}-\psi_{(t)}^{(3)}\right\|=\lim _{m \rightarrow \infty}\left\|\left(S_{m}+T_{m}+I_{m}^{\uparrow}+I_{m}^{\downarrow}\right)^{m} P_{c}^{\prime} \psi-S_{m}^{m} \psi\right\| \leqq e^{\frac{v}{2} t}-1<v t .
$$

Subclaim. $I_{m}^{\uparrow}$ and $I_{m}^{\downarrow}$ are bounded operators, the norm of which is less than a constant, called $v$, times $\frac{1}{2} t / m$.

Proof. $\left\|I_{m}^{\uparrow}\right\|=\left\|\left(1-P_{c}^{\prime}\right) e^{-i e \delta x_{1}^{\prime \prime} t / m} e^{-i\left(H_{B}+e \delta x_{1}\right) t / m} P_{c}^{\prime}\right\|$

$$
\leqq\left\|\left(1-P_{c}^{\prime}\right) e^{-i e \delta x^{\prime \prime} t / m} P_{c}^{\prime}\right\| .
$$

Let $\left(P_{c}^{\prime} \psi\right)(\mathbf{p})=a_{c}^{\prime}(\mathbf{p}) w_{c}^{\prime}(\mathbf{p})$. Introducing the Fourier inverse operator $\mathscr{F}^{-1}$ and $a_{c R}^{\prime}$ the Fourier coefficients of $a_{c}^{\prime}:\left(\mathscr{F}^{-1} P_{c}^{\prime} \psi\right)(\mathbf{x})=\sum_{\mathbf{R}} a_{c \mathbf{R}}^{\prime}\left(\mathscr{F}^{-1} w_{c}^{\prime}\right)(\mathbf{x}-\mathbf{R})$. Then :

$$
\left\|\left(1-P_{c}^{\prime}\right) e^{-i e \tilde{\delta} x_{1}^{\prime \prime} t / m} P_{c}^{\prime} \psi\right\|=\left\|\left(1-P_{c}^{\prime}\right) \mathscr{F} e^{-i e \delta x_{1}^{\prime \prime} t / m} \sum_{\mathbf{R}} a_{c \mathbf{R}}^{\prime}\left(\mathscr{F}-1 w_{c}\right)(\mathbf{x}-\mathbf{R})\right\|^{3}
$$

As $e^{-i e \mathscr{E} x_{1}^{\prime \prime} t ; m}=1-i e \mathscr{E} t / m x_{1}^{\prime \prime} \cdot \theta_{1}\left(x_{1}\right)-\frac{1}{2}(e \mathscr{E} t / m)^{2} x_{1}^{\prime \prime 2} \theta_{2}\left(x_{1}\right)\left(\theta_{1}\right.$ and $\theta_{2}$ are, like $x_{1}^{\prime \prime}$, constant in the intervals $] n a_{1},(n+1) a_{1}[$ and everywhere smaller than one).

$$
\begin{aligned}
\left\|\left(1-P_{c}^{\prime}\right) e^{-i e \mathscr{E} x_{1}^{\prime \prime} t / m} P_{c}^{\prime} \psi\right\| \leqq & e \mathscr{E} t / m\left\|\left(1-P_{c}^{\prime}\right) \mathscr{F} x_{1}^{\prime \prime} \cdot \theta_{1}\left(x_{1}\right) \sum_{\mathbf{R}} a_{\mathbf{c}}^{\prime}\left(\mathscr{F}-1 w_{c}^{\prime}\right)(\mathbf{x}-\mathbf{R})\right\| \\
& +\frac{1}{2}(e \mathscr{E} t / m)^{2}\left\|\left(1-P_{c}^{\prime}\right) \mathscr{F} x_{1}^{\prime \prime 2} \theta_{2}\left(x_{1}\right) \sum_{\mathbf{R}} a_{c \mathbf{R}}^{\prime}\left(\mathscr{F}^{-1} w_{c}^{\prime}\right)(\mathbf{x}-\mathbf{R})\right\| \\
\leqq & e \mathscr{E} t / m\left\|\sum_{\mathbf{R}} a_{c \mathbf{R}}^{\prime}\left(x_{1}^{\prime \prime} \cdot \theta_{1}\left(x_{1}\right)-R_{1} \theta_{1}\left(R_{1}\right)\right) \cdot\left(\mathscr{F}-1 w_{c}^{\prime}\right)(\mathbf{x}-\mathbf{R})\right\| \\
& +\frac{1}{2}(e \mathscr{E} t / m)^{2} \| \sum_{\mathbf{R}} a_{c \mathbf{R}}^{\prime}\left(x_{1}^{\prime \prime 2} \theta_{2}\left(x_{1}^{\prime \prime}\right)\right. \\
& \left.-R_{1}^{2} \theta_{1}\left(R_{1}\right)\right)\left(\mathscr{F}^{-1} w_{c}^{\prime}\right)(\mathbf{x}-\mathbf{R}) \| .
\end{aligned}
$$

We can consider the right-hand side terms as generalised convolutions (see Dunford-Schwartz, Part I, Exercise 3, p. 527), then, for instance:

$$
\left\|\sum_{\mathbf{R}} a_{c \mathbf{R}}^{\prime} \cdot\left(x_{1}^{\prime \prime} \theta_{1}\left(x_{1}\right)-R_{1} \theta_{1}\left(R_{1}\right)\right) \cdot\left(\mathscr{F}^{-1} w_{c}^{\prime}\right)(\mathbf{x}-\mathbf{R})\right\| \leqq \mathscr{M} \cdot\left(\sum_{\mathbf{R}}\left|a_{c \mathbf{R}}^{\prime}\right|^{2}\right)^{1 / 2} \leqq \mathscr{M},
$$

where

$$
\begin{aligned}
& \mathscr{M}=\max \left(\sup _{\mathbf{R}} \int\left|x_{1}^{\prime \prime} \theta_{1}\left(x_{1}\right)-R_{1} \theta_{1}\left(R_{1}\right)\right|\left|\mathscr{F}^{-1} w_{c}^{\prime}(\mathbf{x}-\mathbf{R})\right| d^{3} x,\right. \\
& \left.\sup _{\mathbf{x}} \sum_{\mathbf{R}}\left|x_{1}^{\prime \prime} \theta_{1}\left(x_{1}\right)-R_{1} \theta_{1}\left(R_{1}\right)\right|\left|\mathscr{F}^{-1} w_{c}^{\prime}(\mathbf{x}-\mathbf{R})\right|\right) .
\end{aligned}
$$

3 Here we designate by $x_{1}^{\prime \prime}$ the $x_{1}^{\prime \prime}$-operator in the $x$-representation, i.e., simply, the multiplication operator by $x_{1}-x_{1}^{\prime}$ 
As $w_{c}^{\prime}(\mathbf{p})$ is analytic in the strip $|\operatorname{Im} \mathbf{p}|<A$ (see Appendix), $\left(\mathscr{F}^{-1} w_{c}\right)(\mathbf{x}) e^{A^{\prime}|\mathbf{x}|} \in L^{2} \forall A^{\prime}<A$, then $\mathscr{M}$ exists, furthermore it is small because $x_{1}^{\prime \prime} \theta_{1}\left(x_{1}\right)-R_{1} \theta_{1}\left(R_{1}\right)$ is equal to 0 in the first unit cell, i.e., in the region $\mathscr{F}^{-1} w_{c}$ is large.

A crude estimation of $\mathscr{M}$ will give us:

$$
\mathscr{M}<2\left|\mathbf{a}_{1}\right| \int_{\left|y_{1}\right|>1}\left|y_{1}\right|\left(\mathscr{F}^{-1} w_{c}\right)(\mathbf{y}) d^{3} y \text {. }
$$

Using Cauchy theorem and the fact $\left\|w_{c}^{\prime}\left(\cdot+i \overrightarrow{\mathbf{p}}_{0}\right)\right\|_{L^{2}}=1, \forall\left|\overrightarrow{\mathbf{p}}_{0}\right|<A$ it is possible to choose $A^{\prime}<A$ in such a way

$$
\int_{|\mathbf{y}| \geqq 1}\left|\left(\mathscr{F}^{-1} w_{c}\right)(\mathbf{y})\right|^{2} e^{2 \mathbf{a}_{1} A^{\prime}|\mathbf{y}|} d^{3} y<1 .
$$

Then:

$$
\begin{aligned}
\mathscr{M} & \leqq 2\left|\mathbf{a}_{1}\right| \int_{|\mathbf{y}|>1}\left|y_{1}\right| e^{-\left|\mathbf{a}_{1}\right| A^{\prime}|\mathbf{y}|}\left|\left(\mathscr{F}-1 w_{c}\right)(\mathbf{y})\right| e^{\left|\mathbf{a}_{1}\right| A^{\prime}|\mathbf{y}|} d^{3} y \\
& \leqq 2\left|\mathbf{a}_{1}\right|\left(\int_{|\mathbf{y}|>1} y_{1}^{2} e^{-2\left|\mathbf{a}_{1}\right| A^{\prime}|\mathbf{y}|} d^{3} y\right)^{1 / 2} \# 2\left|\mathbf{a}_{1}\right| \frac{1}{\sqrt{2 A^{\prime}\left|\mathbf{a}_{1}\right|}} e^{-A^{\prime}\left|\mathbf{a}_{1}\right|} \\
& \Rightarrow v T=\frac{2 \pi}{\left|\mathbf{a}_{1}\right|} \mathscr{M} \leqq \frac{2 \sqrt{2 \pi}}{\sqrt{A^{\prime}\left|\mathbf{a}_{1}\right|}} e^{-A^{\prime}\left|\mathbf{a}_{1}\right|}
\end{aligned}
$$

Numerical Estimation of $v T$

$$
\begin{aligned}
& \Delta E_{c^{\prime}} \# 4 \mathrm{eV}=6 \cdot 10^{-19} \mathrm{~J} \text { (gap between the modified conduction band } \\
& \text { and the others) } \\
& \left|\mathbf{a}_{1}\right| \# 5 \AA=5 \cdot 10^{-10} \mathrm{~m}\left(\mathbf{a}_{1} \text { : direct lattice vector }\right) \\
& \mathscr{A}\left(\mathbf{k}_{0}\right) \# 5 \cdot 10^{-7} \Rightarrow A^{\prime} \# 10^{10} \\
& \Rightarrow v T \# 2 \pi \sqrt{2} \frac{1}{\sqrt{A\left|\mathbf{a}_{1}\right|}} e^{-A^{\prime}\left|\mathbf{a}_{1}\right|} \# \frac{4 \pi}{\sqrt{5}} e^{-5}=4 \cdot 10^{-2} .
\end{aligned}
$$

Another estimation can be obtained if we consider the L.C.A.O. approximation [11]. This approximation, valid for a lot of materials, lead us to Wannier functions very near to the atomic ones. For instance, $S$-bands will give us $w_{c}(x) \# e^{-\frac{|\mathbf{x}|}{r_{0}}}$ where $r_{0}$ is the Bohr radius, then

$$
\begin{gathered}
\mathscr{M}<2\left|\mathbf{a}_{1}\right| \cdot \int_{y_{1}>1}\left|y_{1}\right| e^{-\frac{\left|\mathbf{a}_{1}\right|}{r_{0}}|\mathbf{y}|} d^{3} y \# 2 \mathbf{a}_{1} e^{-\frac{\left|\mathbf{a}_{1}\right|}{r_{0}}} \\
\Rightarrow v T=4 \pi e^{-\frac{\left|\mathbf{a}_{1}\right|}{r_{0}}} \# 4 \pi \cdot e^{-10} \# 6 \cdot 10^{-4} .
\end{gathered}
$$

We now give a crude estimate of $\left\|\psi_{t}^{(3)}\right\|=\left\|e^{-i H t}\left(1-P_{c}^{\prime}\right) \psi\right\|$ in :

Theorem 3. $\left\|\psi_{t}^{(3)}\right\| \leqq 2 \ell\left(\Gamma_{c}\right) \frac{1}{\Delta E_{c}} \frac{1}{\Delta E_{c}} e \mathscr{E}\left|\mathbf{a}_{1}\right|$.

Proof. $\left\|e^{-i H t}\left(1-P_{c}^{\prime}\right) \psi\right\|=\left\|\left(1-P_{c}^{\prime}\right) \psi\right\|$.

As

$$
P_{c}^{\prime}=\int_{\Gamma_{c}^{\prime}} \frac{1}{H^{\prime}-z} d z=\int_{\Gamma_{c}^{\prime}} \frac{1}{H_{B}-z}-\int_{\Gamma_{c}^{\prime}} \frac{1}{H_{B}-z} e \mathscr{E} x_{1}^{\prime} \frac{1}{H^{\prime}-z} d z
$$


and $P_{c} \psi=\psi$ we get

$$
\left(1-P_{c}^{\prime}\right) \psi=\left(1-P_{c}^{\prime}\right) P_{c} \psi=\left(1-P_{c}+\int_{\Gamma_{c}^{\prime}} \frac{1}{H_{B}-z} e \mathscr{E} x_{1}^{\prime} \frac{1}{H^{\prime}-z} d z\right) P_{c} \psi
$$

then

$$
\left\|\left(1-P_{c}^{\prime}\right) \psi\right\| \leqq\left\|\int_{\Gamma_{c}^{\prime}} \frac{1}{H_{B}-z} e \mathscr{E} x_{1}^{\prime} \frac{1}{H^{\prime}-z} d z\right\| \leqq \ell\left(\Gamma_{c}\right) \cdot \frac{1}{\Delta E_{c}} \frac{1}{\Delta E_{c^{\prime}}} e \mathscr{E}\left|\mathbf{a}_{1}\right| .
$$

Remark. Taking the same numerical values as above we find that

$$
\left\|\psi_{t}^{(3)}\right\| \leqq 5 \cdot 10^{-10} \mathscr{E} .
$$

\section{Discussion}

In the presence of a periodic potential and an electrical field, we get a resonance phenomena. In fact the preceding calculus show that $\psi_{t}$ is well approximated by the "periodic" function $\psi_{t}^{(1)}$ as long as $t$ is smaller than, say, $10^{2} \mathrm{~T}$ and $\mathscr{E}$ is smaller than $10^{7} \mathrm{~V} / \mathrm{m}$.

To discuss conductivity in crystals it is also necessary to consider the scattering of the electrons by the impurities, the defects and the phonons. In general what has been done is to consider a Boltzmann equation in which the effect of the periodical potential is taken into account by introducing the effective mass of the electron, i.e., to consider it as if was free, but with a different mass. We think it would be interesting to introduce, in the case in which the period, $T$, is larger than the time collision (i.e., in very pure monocrystals at low temperatures and high electric fields), the dynamics that are described in the paper, in the Boltzmann equation.

We expect that the Ohm law, electric current versus electric field, would present a saturation for high fields in these experimental conditions.

\section{Appendix}

As the conduction band is separated from the others by gaps larger than $\Delta E_{c}^{\prime}$, it is easy to prove that $w_{c}^{\prime}$ is analytic in the region $|\operatorname{Im} p|<A$; where $A$ can be calculated in the following manner. $H^{\prime}(k)$ is an analytic family of operators acting on $l^{2}$, in fact we have: $H^{\prime}(\mathbf{k})=H^{\prime}\left(\mathbf{k}_{0}\right)+2\left(\mathbf{k}-\mathbf{k}_{0}\right) \mathbf{K}+\mathbf{k}^{2}-\mathbf{k}_{0}^{2}$. Using the second resolvent equation we obtain

$$
\left(H^{\prime}(\mathbf{k})-z\right)^{-1}=\left(H^{\prime}\left(\mathbf{k}_{0}\right)-z\right)^{-1}\left[1+\frac{1}{2 M}\left(\mathbf{k}^{2}-\mathbf{k}_{0}^{2}+2\left(\mathbf{k}-\mathbf{k}_{0}\right) \mathbf{K}\right)\left(H^{\prime}\left(\mathbf{k}_{0}\right)-z\right)^{-1}\right]^{-1}
$$

from which it is easy to see that as long as

$$
\left\|\frac{1}{2 M}\left(\mathbf{k}^{2}-\mathbf{k}_{0}^{2}+2\left(\mathbf{k}-\mathbf{k}_{0}\right) \mathbf{K}\right)\left(H^{\prime}\left(\mathbf{k}_{0}\right)-z\right)^{-1}\right\|<1
$$

the spectral projector $P_{c}^{\prime}(\mathbf{k})$ and consequently the eigenfunction $\left\{w_{c}^{\prime \mathbf{k}}(\mathbf{K})\right\}$ are analytic in $\mathbf{k}$.

Condition (A) is satisfied if

$$
\left|\frac{1}{2 M}\left(\mathbf{k}^{2}-\mathbf{k}_{0}^{2}\right)\right| \frac{1}{\operatorname{dist}\left(z, \sigma\left(H^{\prime}\left(\mathbf{k}_{0}\right)\right)\right)}+\frac{1}{M}\left|\mathbf{k}-\mathbf{k}_{0}\right|\left\|\mathbf{K}\left(H^{\prime}\left(\mathbf{k}_{0}\right)-z\right)^{-1}\right\|<1 .
$$


The second term in the left-hand side can be estimated using again the resolvent equation:

$$
\begin{aligned}
\left\|\mathbf{K}\left(H^{\prime}\left(\mathbf{k}_{0}\right)-z\right)^{-1}\right\| \leqq & \mid \mathbf{K}\left(\frac{\mathbf{1}}{2 \mathrm{M}}(\mathbf{k}+\mathbf{K})^{2}-\xi\right)^{-1} \| \cdot\left(1-\left\|V^{\prime}\left(\frac{1}{2 M}(\mathbf{k}+\mathbf{K})^{2}-\xi\right)^{-1}\right\|\right)^{-1} \\
& \cdot\left(1+\frac{|z-\xi|}{\operatorname{dist}\left(z, \sigma\left(H\left(\mathbf{k}_{0}\right)\right)\right)}\right) \leqq \sup _{\mathbf{K}}\left|\frac{\mathbf{K}}{\frac{1}{2 M}(\mathbf{k}+\mathbf{K})^{2}-\xi}\right| \\
& \cdot\left[1-\left(\sum_{\mathbf{K}}\left|V_{\mathbf{K}}^{\prime}\right|^{2}\right)^{1 / 2}\left(\frac{\left.\sum_{\mathbf{K}} \frac{1}{2 M}\left(\mathbf{k}_{0}+\mathbf{K}\right)^{2}-\xi\right)^{2}}{\mid}\right)^{1 / 2}\right]^{-1} \\
& \cdot\left(1+\frac{|z-\xi|}{\operatorname{dist}\left(z, \sigma\left(H\left(\mathbf{k}_{0}\right)\right)\right)}\right) \equiv \mathscr{A}\left(\xi, \mathbf{k}_{0}\right) .
\end{aligned}
$$

As $\xi$ is, in a large extent, arbitrary, the only condition it has to satisfy is to be sufficiently negative so that $\left(\sum_{\mathbf{K}}\left|V_{\mathbf{K}}^{\prime}\right|^{2}\right)^{1 / 2}\left(\sum_{\mathbf{K}} \frac{1}{\left(\frac{1}{2 M}\left(\mathbf{k}_{0}+\mathbf{K}\right)^{2}-\xi\right)^{2}}\right)^{1 / 2}<1$; we choose it in such a way to minimize $\mathscr{A}\left(\xi, \mathbf{k}_{0}\right)$ and call $\mathscr{A}\left(\mathbf{k}_{0}\right)=\inf _{\xi} \mathscr{A}\left(\xi, \mathbf{k}_{0}\right)$. So (B) is satisfied if $\mathbf{k}$ is such that

$$
\frac{1}{2 M}\left|\mathbf{k}^{2}-\mathbf{k}_{0}^{2}\right| \frac{1}{\Delta E_{c}^{\prime}}+\frac{1}{M}\left|\mathbf{k}-\mathbf{k}_{0}\right| \mathscr{A}\left(\mathbf{k}_{0}\right)<1
$$

i.e., if $\mathbf{k} \in \mathbb{C}$, belongs to a certain ball $\mathscr{B}_{\mathbf{k}_{0}}$, centered in $\mathbf{k}_{0}$. Then the region of analyticity of $\left\{w_{c}^{\mathbf{k}}(\mathbf{K})\right\}$ is $\bigcup_{\mathbf{k}_{0} \in \mathbb{B}} \mathscr{B}_{\mathbf{k}_{0}}$, or in other words $A$ is the minimum over $\mathbf{k}_{0} \in B$ of the radius of the balls $\mathscr{B}_{\mathbf{k}_{0}}$.

Acknowledgements. I would like to thank E. Lieb and B. Simon for their kind hospitality at Princeton. The author is very grateful to J. Ginibre for pointing out an error in an Appendix.

\section{References}

1. Kittel, C.: Quantum theory of solids. New York: Wiley 1964

2. Wannier, G.H.: Elements of solid state theory. Cambridge: Cambridge University Press 1959

3. Adams, E.N. : Phys. Rev. 107, 698 (1957)

4. Avron, J., Grossmann, A., Rodriguez, R.: Rept. Math. Phys. 5, 113 (1974)

5. Avron, J., Grossmann, A., Rodriguez, R., Zak, J.: Ann. Phys. 103, 47 (1977)

6. Bentosela, F.: Thesis. Université Aix-Marseille 2 (1977)

7. Reed, M., Simon, B.: Methods of modern mathematical physics, Vol. IV. New York: Academic Press 1978

8. Wilcox, C.: Technical summary report no. 32. University of Utah (1977)

9. Thomas, L.: Commun. Math. Phys. 33, 339 (1973)

10. Avron, J., Simon, B.: Ann. Phys. 110, 85 (1978)

11. Bassani, F., Parravicini, G.: Electronic states and optical transitions in solids. Pergamon Press (1975)

Communicated by J. Ginibre

Received June 30, 1978; in revised form March 10, 1979 